the day the boy died, and these revealed a copious invasion both of the blood and cerebro-spinal fluid by a variety of streptococcus, probably longus, according to Dr. Horder.

We come now to the necropsy made on April 26th, an account of which I will read to you. By the light thrown upon the case after death we can readily see that we were face to face with most of the symptoms now recognized as depending on toxaemia due to an arterial pyaemia starting from the mitral valve of the heart-to wit, pyrexia, haemorrhagic effusions, and visceral infarctions. Clinically there are several types of the malady, for it is apt at times to simulate enteric fever, acute general tuberculosis, and, as in some respects in this instance, even typhus fever. Hence the diagnosis is by no means always easy to make till some time has elapsed.

Necropsy Twenty Hours after Death.

Well-grown, fairly-nourished boy. Many small petechial haemorrhages, specially over trunk and lower arms.

Head.-Cranial bones natural; a few subpericranial haemorrhages. Dura mater and sinuses natural. Arachnoid and pia mater: Over convexity a few small subarachnoid haemorrhages. Arteries : Larger arteries natural. Brain $54 \mathrm{oz}$. Puncta cruenta well marked. Spinal cord not examined Eyes not examined. Ear: In right ear some blood-stained mucus; left natural.

Chest.-Oesophagus: A number of small subepithelial haemorrhages throughout length. In lateral wall of pharynx just behind the tonsi on either side an area the size of a shilling covered with thin yellow granular "membrane." Glands in neck natural. Larynx and trachea: A number of snall petechiae. Lungs: Right $170 z$ left $8 \mathrm{oz}$. A few small subpleural haemorrhages on either lung. Both lungs congested. Heart, $8 \frac{1}{2} \mathrm{oz}$. A drachm or two of slightly turbid fluid in pericardial sac. Beneath visceral pericardium a few petechiae. Heart muscle pal with a few minute haemorrhages in its substance. Pulmonary, tricuspid, and aortic valves natural. The mitral valve was completely surrounded as to its free edge with a ridge of recent granulations about $\frac{1}{3}$ in. wide and $\frac{1}{6}$ in. high. The granulations were yellowish and soft in consistence. Aorta and vessels natural.

Abdomen.-Peritoneum natural. Stomach: Many small petechiae in mucous membrane. Intestines, a few petechiae. Liver, $54 \mathrm{oz}$; very pale, large, no haemorrhages. Spleen, ro oz., soft; two or three recent medium-sized infarcts. Pancreas natural. Abdominal lymphatics natural. Suprarenals natural. Kidneys, $6 \mathrm{oz}$. each. On the surface and in the substance of each were innumerable small haemorrhages. In the right one moderate-sized anaemic infarct and another larger recent one, of which only the middle third was anaemic, the outer third on either side of the central wedge being haemorrhagic. In the left kidney a similar appearance externally with another large haemorrhagic infarct. Ureters and bladder natural. Organs of generation natural. Joints natural.

Nature of Disease.-Endocarditis, malignant. Splenic and renal infarcts. Petechial haemorrhages in skin, all serous membranes, oesophagus, stomach, and trachea.

We now learned the cause of all the severe symptoms, and may fairly ask why we did not determine the true nature of the malady during life. I suppose the answer is that we were chiefly migled in consequence of finding no plain indications of endocarditis as evidenced by a cardiac murmur. I think we should have had no hesitation in our diagnosis if there had been a constant or a varying murmur. You will remember that Dr. Gould heard a murmur on the day of the boy's admission, and again three days later, but this was fleeting, and never heard again by me or by any of the clinical clerks. Yet there was another indication of cardiac disease. The heart's apex was a little displaced. Again, the condition of the spleen might have suggested infarctions, and had there been haematuria, which, strange to say, was absent, despite the haemorrhages and infarctions found in the kidneys, we might have been set on the right track. I take up this point, and consider it a little further. It is sufficiently recognized that the malady this boy suffered and died from sometimes runs its course without the occurrence of cardiac murmurs, and this fact must always add serious]y to the difficulty of making a certain diagnosis in such a case. Yet we have to learn to suspect an endocarditis in the absence of valvular murmurs. This is one lesson. We have nothing in this boy's history pointing to any previous disease of his mitral valve. It is therefore very noteworthy that the lad was seemingly in good health till the day he sickened, and his illness became at once grave. We may next inquire as to the source of the streptoroccal invasion. In many of these cases there is reason to believe that such infection occurs in the lymphatics of the throat, but there remains room here for further research into these matters. There was some granular membrane found in the pharynx in this case, as we have seen. We may take note again of the absence of signs of endocarditis in these cases, and bear the fact in mind when we are dealing with cases of obvious rheumatic fever and of chorea. Here, again, we are in the presence of an infection and a toxaemia, due also to a member of the atreptococcal family, and we pay careful attention to the state of the heart and its several textures, are always on the alert to detect the earliest signs of inflammatory mischief, and always glad to find no evidence of it. But may we not sometimes be wrong in concluding that the absence of murmurs is a certain sign of immunit of the endocardium in such instances? I am inclined to think we are sometimes misled in this matter, and that such inflammatory changes actually exist without giving token of their presence, provided they are small, bead-like, and not grossly vegetative. These, then, are the chief lessons of this sad case. I think nothing further could have been done for this patient, or for any in like case when the malady is so virulent and overwhelming. Happily, there are degrees of intensity of all forms of toxaemia. Some of these cases of infective endocarditis last for months and even for 8 year. I have had such cases under my observation. In the chronic forms we now seek, as you know, to introduce antitoxic sera into the system by the skin or by the bowel, and sometimes with success. We may look forward to better results when the presence of particular strains of micrococei are recognized in the blood, and specific sera are manufactured to combat these. Hitherto there has generally been \& negative result in blood cultures from these patients, but $\mathrm{Dr}$. Horder tells me he believes this is often due to too small an amount of blood being dealt with. A finger-prick or ear-prick does not yield sufficient for an adequate examination, and blood should be taken from a brachial vein with due precautions. Dr. Kanthack was wont to say that if in any case the blood contained toxic particulate elements, the prognosis was unfavourable. We may now, however, hope for better results in the near future, and so be enabled to render less grave the prospects of this terrible malady.

\section{A Clintiral}

\section{EARLY MICROSCOPICAL DIAGNOSIS OF TUMOURS.}

Delivered at St. Bartholomew's Hospital on June 29th.

BY C. B. LOCKWOOD, F.R.C.S., Surgeon to the Hospital.

I NEED not dwell for one single instant upon the importance of the early diagnosis of tumours. In the beginning the whole disease may be taken away with an abundant margin for safety; towards the end who can say whither it may not have spread?

Disabuse your minds of much of that which you have read or been told.

In books tumours are "innocent" or " malignant," but in Nature such distinctions do not exist. In a little while I shall tell you of tumours which seemed to be innocent and yet were malignant, and of tumours which seemed malignant and yet were innocent. Do not be misled by the positive air wherewith some assert the nature of tumours which they cannot see and have only felt with difficulty. The errors they fall into would be ludicrous were they not fraught with tragedy. It is, moreover, strange to see how soon these errors are for $^{2}$ gotten. It is, however, an attribute of the human mind to remember the agreeable and forget the disagreeable. After guessing it is pleasant to be right; unpleasant to be wrong so that the right guesses are remembered and paraded and the wrong forgotten.

I, too, once thought that the rules of art would enable me to tell clinically whether a tumour was innocent or malignant, but much has happened to shake my confidence. Some years ago I was asked to see a patient and remove an abdominal tumour. The abdomen of this unfortunate woman contained ascitic fluid in abundance, and masses of malignant disease. The liver, too, was enlarged and full of nodules. Naturally I looked for the primary growth. In theleft breast was a globular tumour about an inch in diameter, and the axillary and subclavian lymphatic glands were enlarged. Sixteen years before a small cyst had been found in the left breast at the site of the tumour. The cyst had been tapped and injected with iodine. A lump remained and had slowly but steadily grown. As you are aware, cysts of the mammary gland sometimes contain an intracystic tumour growing from their walls. In the case which has just been narrated it was almost certain that the intracystic growth was sarcomatous. Those of jou who are 
very rightly cultivating your critical faculties may have thought me guilty of a flagrant inconsistency, for, as you may remember, 1 was very positive about the diagnosis of the abdominal tumours which I had not seen. But my theme is the early diagnosis of tumours. It is in their earlier stages that their diagnosis is impossible by ordinary clinical methods; in their later stages their diagnosis is easy, but by that time they are often beyond the reach of surgery. In its beginning no one could have diagnosed the mammary tumour by ordinary clinical methods. Every one might have known that it was a cyst, but no one that the cyst contained sarcoma until after its removal and examination with the microscope. Owing to improvaments in surgery, a cyst would nowadays be unhesitatingly 1 woved. The dangers of the operation would be inappreciable and the subsequent suffering comparatively slight. But who can tell the happiness of the patient to be freed from the sense of impending evil ?

Here is another incident which should shatter your faith in the infallibility of the mere clinical diagnosis of commencing tumours; and remember it is futile to diagnose them when far advanced.

A girlish-looking woman, aged 29. had a small, hard, movable tumour in the right breast. On the strength of the clinical history and examination, it was said to bean innocent tumour-a fibro-adenoma-and the house-surgeon kindly removed it. It was sent to the pathological laboratory for examination and report. The pathologists pronounced it to be a cancer, and sent me a section which left no room for doubt. After consultation with my colleagues I removed the whole mammary gland, pectoral muscles, and axillary fat and lymphatics. My reasons for this severe operation were: first, a mere limited removal of a carcinoma is useless, because other islands of cancer may be present in the mammary gland. In this instance more cancer was found near the site of the original tumour. Secondly, the pectoral muscles were removed lest they, too, should contain carcinoma, and above all to give access to the lymphatics along the course of the axillary vein and of its main branches. Some of the axillary lymphatic glands were enlarged, although, as is the rule in the early stages, none could be felt. This patient was well two years after the operation. She was more fortunate than another young woman of 28 , whose carcinomatous breast was removed in the same way. Hers relapsed within two years, but it was far advanced at the time of operation. She had lost one of her best chances of safety.

There is something rather mysterious about the invasion of the lymphatics in malignant disease. The other day a microscopical examination disclosed no carcinoma in the enlarged axillary glands, but plenty in others which were too small to be felt. I use the term malignant in order to include the sarcomas. There seems to be an idea abroad that sarcomas do not invade the lymphatics. I cannot think how such a gross error can prevail. In my experience the sarcomas do invade the lymphatics. And this fact ought always to be taken into consideration just as in cases of carcinoma. As a diagnostic indication the supposed presence or absence of lymphatic enlargement is not of much value. In stout women the lymphatic glands upon and beneath the costo-coracoid membrane cannot be felt although greatly enlarged, and the same remark applies to those in the axilla

I would also advise you to be very sceptical as regards the naked-eye characters of young tumours. Had that carcinoma of the breast been judged by the naked eye a wrong conclusion would have been arrived at, and the patient would not have been as safe as she is. Over and over again I should have been wrong had I relied on the mere naked-eye characters of young tumours; sometimes the whole breast would have been removed for mastitis, and cometimes the breast would have been left with cancer in it. In their later and more hopeless stages the character of tumours can be roughly judged with the naked eye. For this purpose a complete incision through the tumour and its margins is necessary. You know how a malignant tumour grows into its surroundings. But do not be deceived by the presence of a capsule. I have removed a carcinoma of the breast which had a perfect capsule. A pedicle, too, may deceive especially in growths within the urinary bladder. I am sure that it is rash to rely upon the supposed innocency of a tumour. Recently you saw me with infinite labour remove a huge tumour growing from the horizontal ramus of the pubes on the right side. The stories told by patients are to be received with incredulity, but in this instance we had reliable medical evidence. The growth had grown very slowly for eighteen years, but for six months had increased rather rapidly. During the operation some enlarged lymphatic glands were found near the tumour, and others were felt enlarged upon the opposite side of the pelvis. The pathological report, with which I agree, says that " the cartilaginous portion is very cellular, suggesting malignant disease." The gland contains what I believe to be sarcoma. Now whatever may ultimately accrue, you will agree with me that the later stages of this so-called innocent tumour were most forbidding. Do not trust innocent tumours, remove them early. I have recently operated for carcinoma of the breast which had been seen by a medical man two years before. The tumour was adherent and on the point of ulceration. Unfortunately, such procrastination is not infrequent. Perhaps it is a survival of the days when there, were no anaesthetics and no antiseptics.

There is some strange relationship between tumours which are labelled innocent and those which are labelled malignant. In April, 1899, I removed a small cystic fibro-adenoma from the right breast of a woman aged 45. At this time some of the lymphatic glands in the axilla could be felt slightly enlarged but soft and movable. In April, 1904, I removed the left breast, pectorals, and axillary lymphatics for carcinoma. Clinically the carcinoma was a small, hard, movable nodule, exceedingly like the cystic fibro-adenoma removed five years before. The lymphatic glands in the right axilla were still to be felt, and were perhaps larger than at the first operation. Some would call this occurrence a mere coincidence, but that would not convey much to a rational mind.

The fallacies and difficulties which pertain to the clinical and naked-eye diagnosis of young tumours are easily overcome by examining them with the microscope as soon as possible after their discovery. I strongly advise you to dismiss from your minds any lingering belief you may have in diagnostic infallibility and adopt this rule.

Nearly the whole of the examinations which I am about to narrate were made with the help of Mr. Ernest Shaw, to whom I am much obliged. The procedure is quite simple, but requires practice and a considerable experience of the morbid histology of tumours. When the patient has been anaesthetized an incision is made and a portion of the tumour is cut off and frozen on a Williams ether-freezing microtome. Sections are then cut and floated upon a slide, and stained with a solution of methylene blue, protected with a cover-glass, and examined.

Mr. Shaw has been able to show me exceedingly good sections within the space of five to seven minutes. ${ }^{1}$ Now and then a second piece of the tumour has been removed and examined. Presently you shall hear the results of our essays, but first I would refer to the obvious advantages of this method. Every one has very naturally a dread of anaesthetics. Besides the minute danger which attends their administration, it cannot be denied that they usually make the patient very sick and ill. Indeed, I cannot help considering our present methods of anaesthesia as being exceedingly crude, even in the most skilful hands. Well, the immediate histological examination of the tumour obviates the necessity for a second anaesthesia, with ali its attendant discomforts and anxieties. Next, it is most reassuring to the patient to know that nothing will be left to chance. They become resigned when they reailize that should the microscopical examination prove the tumour to be innocent it alone will be removed; but should the mieroscopical examination prove the tumour malignant, then whatever is necessary for ultimate safety can be at once accomplished. For my own part, I may say that I can proceed with greater confidence and persistency throughout a prolonged operation for malignant disease when I have clearly seen the cancerous tumour under the microscope. Not long since a small nodule in the breast of a very stout woman was seen to be colloid carcinoma, and this permitted of no half measures.

I also think the surgeon ought to see and judge for himself. In such an important matter the surgeon himself ought to be able to rely upon his own judgement. Some years since an elderly lady had an ulcer about an inch in diameter to the right of the urinary meatus. The clinical characters of the ulcer were dubious. The first two sets of frozen sections were pronounced by others to be inflammatory. After having seen a third I was convinced that the nlcer was an epithelioma of the most malignant type, and thereupon performed an extensive operation for its removal. Unfortunately this attempt was unavailing, for after the lapse of a few months the disease reappeared near the same spot, and a second attempt was equally unsuccessful.

It is obvious that differences of opinion may arise as to the correct interpretation of microscopical sections, and in that 
case the surgeon should be prepared to accept the responsibility of deciding. A woman was sent with a hard tumour in the right breast, retraction of the nipple, and enlarged axillary glands. The tumour was adherent to the skin and to the pectoral muscles, and it looked like a desperate case of carcinoma. There was, however, one ray of hope. An ulcerated opening near the retracted nipple discharged a little thick, yellow, odourless pus, not at all like the thin blood-stained, offensive discharge from a cancerous ulcer. I concluded, although others were doubtful, after the immediate microscopical examination that the tumour was tuberculous. The removal of the mammary gland and of the enlarged lymphatics was followed by a speedy recovery.

The value of an immediate histological examination was exemplified under the following difficult circumstances: A lady had a sanious discharge from an enlarged uterus, behind which a hard tumour could be felt in Douglas's pouch. In addition her abdomen was distended by a fluid swelling. It was difficult to say whether the fluid was all free or whether some of it was circumscribed. The question of carcinoma of the uterus was mooted and the assumption of its presence seemed to explain the symptoms. An unavailing attempt had been made to diagnose the condition with the aid of an anaesthetic. The patient was very averse to take another anaesthetic to obtain a specimen for a postponed histological examination, to be followed, perhaps, by a third anaesthesia. She agreed at once to take an anaesthetic to allow of an immediate microscopical diagnosis of the uterine condition, to be followed up by an abdominal operation if the result was favourable. Clearly, if the uterus was carcinomatous, it was of little use exploring the abdomen. Assisted by Dr. Hope Murray and Dr. Bolus I dilated and curetted the uterus. Thesubstanceremoved was chronically inflamed endometrium. I then opened the abdomen. It was full of blood which continued to gush out freely. At first the flow was very darkcoloured; as though it had been long effused, but afterwards it became bright red. Guessing that this had escaped from a ruptured ovarian cyst, the hand was thrust into the pelvis and seized the pedicle of a ruptured ovarian cyst full of papillomatous growth which bled freely. After the cyst had been taken away, a large pedunculated fibroid had to be removed from the back of the uterus to allow of an inspection of the right ovary, which was healthy. The patient made a good recovery and remains well.

An immediate histological diagnosis was the prelude to an operation for the removal of gall stones. A lady had symptoms which pointed to cholelithiasis, but she had in addition most suspicious tumours in both breasts. Obviously if these tumours were carcinomatous it would not be worth while to begin with the gall bladder. I arranged to give an anaesthetic and effect an immediate microscopical examination of the tumours of the breasts, and if the proved to be innocent proceed forthwith to explore the gall bladder. In a few minutes we saw under the microscope very perfect sections of a chronically-inflamed mammary gland. The gall bladder was then emptied of a quantity of stones, and the mammary tumours slowly disappeared. These inflammatory tumours of the breast are by no means infrequent. Under the microscope they are easy to distinguish from carcinoma because the inflamed lobules and the little islands of gland tissue possess easily-seen branching ducts. This feature was clearly seen in a recent case, in which a cyst was likewise found in the centre of the inflammatory mass. Before the immediate microscopical examination, and after the naked-eye inspection of the cut surface of the mass, I myself would have given a verdict in favour of carcinoma. I wonder bow often those who trust to the naked eje have mistaken chronic mastitis for carcinoma.

Chronic mastitis and carcinoma may co-exist. Two years ago great differences of opinion prevailed at consultations as to the nature of tumours in both breasts of a young woman whom I showed to my colleagues. On the right side one of the tumours felt a little harder than the rest and was ultimately found to be a carcinoma.

The immediate method of microscopical diagnosis is also applicable to tumours of the tongue. For instance, a gentleman had a small chronic ulcer upon the edge of the tongue. Its base was very hard and an enlarged lymphatic gland could be felt deeply situated beneath the jaw. Usually a piece of such a tumour is removed with the aid of cocaine, and prepared for postponed microscopical diagnosis. This method does not give very certain results, because the piece removed is sometimes too small, and does not include a sufficient depth of the base of the ulcer, which, after all, is the essential part. Now two points had to be considered with regard to this ulcer: first, if it were an epithelioma a wide removal was necessary for future safety; and, secondly, the gland beneath the jaw ought also to be removed, necessitating an incision in the neck. A general anaesthetic was given and a complete section of the ulcer showed that although no cell nests were present, nevertheless the most suspicious epithelial ingrowth was in progress, accompanied with much small-celled infiltration. After this had been seen the ulcer was freely removed, and afterwards the gland beneath the jaw was taken away, together with another which was unexpectedly encountered. As you are aware, we frequently remove suspicious lymphatic glands for postponed microscopical examination. A gland removed from the neck of a man who looked as though he had inoperable malignant disease proved to be tuberculous. An opportunity occurred of seing whether it was possible to ascertain the nature of enlarged glands by the immediate method. A woman with a very advanced ulcerating carcinoma of the left breast had an enlarged lymphatic gland nearly at the apex of the posterior triangle of the neck. A few minutes after it had been removed we saw that it contained carcinoma. Afterwards, at this woman's earnest entreaty, I removed the breast and pectoral muscles, and all the lymphatics from the apex of the posterior triangle of the neck down to the axilla. She made a very rapid recovery.

If desirable the characters of abdominal tumours can be immediately ascertained during the progress of the operation. A piece removed from a tumour of the liver proved to be a cylindrical-celled carcinoma. The primary growth was in the large intestine. A piece removed from the vicinity of a tumour growing within the small intestine proved to be inflammatory, but no more could be obtained for fear of a faecal leak. The tumour itself was not removed because the naked-eye inspection made us all quite sure that the tumour and the enlarged lymphatic glands were malignant. However, this guess was wrong, for the large tumour speedily disappeared, and not a trace of it could be discovered when the abdomen was examined in the post-mortem room. The patient died some weeks after the exploration from the effects of septic inflammation of the heart, pericardium, and lungs. The original seat was never discovered. Such cases as these explain the disappearance of many supposed malignant growths.

It is sometimes difficult to tell during the course of operations for the removal of tumours whether all the disease is being removed. For tumours of the breast the nitric acid method of Harold J. Stiles ${ }^{2}$ gives instructive results. Few realize either the extent of the mammary gland itself or the ramifications of its carcinomata until they have tried Stiles's method. But for other kinds of tumours it is not applicable. Nevertheless, the question not infrequently arises during the removal of tumours whether the incision has been carried into healthy tissues. Here the immediate method of microscopical examination tells at once what we wish to know. Whilst removing that huge ossifying chondro-sarcoma about which I have told you, a doubt arose as to the nature of some of the tissues in its vicinity. A microscopical section was made forthwith, and showed that the suspected part was merely. fibrous tissue.

This simple method of immediate microscopical examination ought to be particularly useful for the diagnosis of testicular tumours. Nowadays the testicle has ofttimes to be removed, and then a postponed microscopical examination made. It is disconcerting when the tumour proves to be a gumma, which, as you know, can be cured by a course of potassium iodide and mercury. Doubtless this element of uncertainty has hitherto been a fair excuse for delay; but, whilst delaying, the growth may have invaded the spermatic cord, the lymphatics within the abdomen, or lungs, or other organs. Patients would submit to early operation if they felt sure that it would not lead to an unnecessary mutilation. The following cases illustrate the difficulties of the diagnosis of testicular tumours.

A young man, aged 24 years, had a small hard lump in the right epididymis. A senior surgeon of great experience at once concurred in the opinion that this was tuberculous and advised castration. After removal the section through the tumour showed many jellow opaque areas, also cysts, and growth which looked medullary. The microscope revealed a variety of carcinoma which is sometimes called malignant adenoma. To me the sections looked like the Wolffian body of an embryo pig or rabbit. Similar growths are met with in the kidney, and doubtless have a similar origin from the Wolffian body. Six years afterwards this patient had the opposite testicle removed for a malignant tumour. 
On another occasion a patient was seen with a small elastic swelling near the head of the right epididymis. It felt like one of the cysts which are so commonly met with in that situation, but it was not translucent. A small trochar and cannula were used to empty the supposititious cyst, butonly a little blood ran out, and the lumen of the trochar was partially blocked with a scrap of soft tissue. This was pronounced by the pathologist to be sarcomatous. The testicle was removed and a small sarcomatous tumour found in the body of the testis close to the head of the epididymis.

In conclusion, you will remember when confronted with a young and ambiguous tumour to assume an attitude of intel. lectual humility, and carefully eschew diagnostic omniscience and infallibility.

For knowledge is of things we see.

NOTE AND REFERENCE.

1 I am told that Mr. Shaw has been timed to have the section ready in two minutes. 2 The Surgical Anatomy of the Breast and Axillary Lymphatic Glands, Edinburgh Medical Journal, June and July, 1892.

\section{CONCERNING PADS UPON THE FINGER JOINTS AND THEIR CLINICAL RELATIONSHIPS.}

By AŔCHIBALD E. GARROD, M.A., M.D., F.R.C.P., Assistant Physician, 8t. Bartholomew's Hospital; Physician, Hospital for Sick Children, Great Ormond Street.

THE lesions which form the subject of the present communication are not in themselves serious, but it is desirable that those who practise medicine should be familiar with them, if only that they may distinguish them from other and more serious troubles which they simulate to some extent.

The pads in question formed the subject of a short paper, based upon the observation of three cases, which appeared in the volume of the St. Bartholomew's Hospital Reports for the year 1893 (vol. xxix, p. 157). Since that time I have met with other cases in which such pads or nodules were present, and as these additional cases throw some further light upon their clinical relationships, I venture to return to the subject.

They form excrescences which are almost confined to the dorsal aspects of the interphalangeal joints of the proximal row, and are only very rarely seen upon the terminal joints of the fingers. They are usually present upon the fingers of both hands, but in their distribution there is no striking symmetry. They vary considerably in size, and may be no larger than split peas, or may be as large as the halves of hazel nuts. Although usually central in position, they may incline to one or other side of the joint.

When the fingers are extended the pads are moderately soft, and can be moved, to some extent, upon the underlying structures, but when the fingers are flexed they become firm and hard and are no longer movable.

There are no indications of disease of the joints over which they lie, and such pain as is felt is referred to the pads rather than to the joints themselves. In a radiograph picture (see figure) the bones appear normal as also do the joints, but the

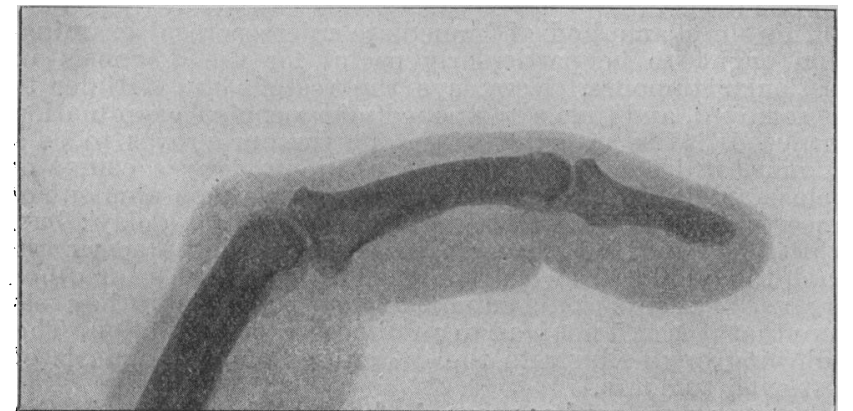

central parts of the pads are seen to be slightly less pervious to the Roentgen rays than the remainder of the soft structures of the fingers.

Sometimes the lumps are quite painless, but more often pain, of various degrees of severity, is complained of, especially when the fingers are flexed, and tenderness is elicited by the slight traumatisms which are unavoidable in daily life. Occasionally the development of them is attended by more severe pain, which may even shoot up the arm as high as the elbow.

The pads may make their appearance at any period of life, and when once formed they appear to be permanent. Patients who exhibit multiple pads usually state that they did not form simultaneously but at intervals. The growth is usually rapid and may occupy as short a period as a few weeks. In some cases the formation of each pad is attributed to some slight injury, such as may result from impact against a piece of furniture or a blow from a cricket-ball. In other instances no such cause is assigned.

I have had no opportunity of examining such a pad post mortem, and am therefore unable to give any account of their structure, but their clinical features recall those of thickened bursae, and it is difficult to doubt that they are mainly composed of fibrous tissue.

Of twelve cases of which I have notes, seven were in males and five in females. In five cases the pads appeared before the patients had reached the age of 20 years, and in three of these at an earlier age than 15 years. On the other hand, in one case they developed at the age of 60 years. In one case they were said to have been present for 42 years. Three patients gave clear histories of similar lesions in near kinsfolk.

The pain and swelling in the neighbourhood of the fingerjoints is apt to excite apprehension of further developments, but there does not appear to be any connexion between these pads and any of the morbid conditions which are usually grouped together under the names of rheumatoid or osteoarthritis. It should be mentioned, however, that two elderly patients had well-marked Heberden's nodes in addition to the pads.

Of the three cases described in 1893, the pads were associated with Dupuytren's contraction of a finger in one, and the father of a second patient was said to have that deformity

The association thus suggested is much more clearly brought out by the larger number of cases now available, and it seems impossible to doubt there is an intimate connexion between the two conditions, or that they have a common cause. On the other hand, it is clearly the case that the pads usually develop at a much earlier period of life than does the contraction of the palmar fascia.

Of the twelve patients no less than six themselves exhibited Dupuytren's contraction. In most instances the puckering in the palm was well marked and the contraction was quite of the ordinary kind, but in one or two there was merely a fibrous thickening on the palmar aspect of a little finger, causing flexion, but without any obvious implication of the palmar fascia.

The fathers of three other patients, and also of one of the six already referred to, were said to have fingers so drawn down, and in one of the three remaining cases the occurrence of Dupuytren's contraction in the family was not inquired into. Thus in no less than nine out of the twelve cases there was a parental or personal history of such contraction, and the association in solarge a proportion of the cases of two lesions, neither of which can be described as common, can hardly be fortuitoüs.

Dupuytren's contraction, although hardly a gouty lesion, is usually held to be connected with gout. It might perhaps be styled a para-gouty lesion. It is therefore of interest to consider the history of the twelve patients in this connexion. Only one patient had himself suffered from attacks of articular gout. In four cases there was a definite history of true gout in direct ascendants, in five the history was doubtful on this point, and in the remaining five neither family nor personal history of gout was forthcoming. The connexion with gout is clearly less obvious than that with Dupuytren's contraction:

I know of no plan of treatment which is of any avail in reducing the size of the pads or in causing them to disappear, and it is seldom that the pain and inconvenience which they cause is very serious. Beyond the fact that a patient who develops them in early life appears to be liable, as time goes on, to develop Dupuytren's contraction of the palmar fascia, they do not seem to possess any grave prognostic significance.

Bequests to Hospitals.-Under the will of the late $\mathrm{Mr}$. William Riley, of Leamington and Birmingham, who died on May ist, a sum of $£ 3,050$ is appointed to be held in trust, as to $£ 800$ for the Warneford Hospital, Leamington, as to $£ \mathrm{I}, 000$ for the Queen:s Hospital, Birmingham, and as to $£_{1}, 250$ to the General Hospital, Birmingham, in each case for the same purpose, namely, the establishment of a bed to be called after the testator. 